\title{
A Crisis-Responsive Framework for Medical Device Development during the COVID-19 Pandemic
}

Antonini, Marc-Joseph 1,2,3,4,t; Plana, Deborah ${ }^{1,4,5,+}$; Srinivasan, Shriya ${ }^{1,6,7,8, t ;}$ Atta, Lyla ${ }^{1,9}$; Achanta, Aditya ${ }^{1,10}$; Yang, Helen ${ }^{1,11}$; Cramer, Avilash ${ }^{1,4}$; Freake, Jacob ${ }^{1,12}$; Sinha, Michael S. 1,11; Yu, Sherry H. 1,13; LeBoeuf, Nicole R. ${ }^{1,14}$; Linville-Engler, Ben ${ }^{1,15,16,{ }^{*} ; \text { Sorger, Peter K. 1,5,11, }{ }^{*}, \neq}$

${ }^{1}$ Greater Boston Pandemic Fabrication Team (PanFab) c/o Harvard-MIT Center for Regulatory Science, Harvard Medical School, Boston, MA, USA

${ }^{2}$ Research Laboratory of Electronics, Massachusetts Institute of Technology, Cambridge, MA, USA

${ }^{3}$ McGovern Institute for Brain Research, Massachusetts Institute of Technology, Cambridge, MA, USA

${ }^{4}$ Harvard-MIT Division of Health Sciences \& Technology Program, Cambridge, MA, USA

${ }^{5}$ Harvard Ludwig Cancer Research Center and Department of Systems Biology, Harvard Medical School, Boston, MA, USA

${ }^{6}$ Department of Mechanical Engineering, Massachusetts Institute of Technology, Cambridge, MA 02139, USA

${ }^{7}$ Division of Gastroenterology, Hepatology and Endoscopy, Brigham and Women's Hospital, Harvard Medical School, Boston, MA 02115, USA

${ }^{8}$ David H. Koch Institute for Integrative Cancer Research, Massachusetts Institute of Technology, Cambridge, MA 02139, USA

9 Johns Hopkins University School of Medicine, Baltimore, MD, USA

${ }^{10}$ Harvard Medical School, Boston MA, USA

${ }^{11}$ Harvard-MIT Center for Regulatory Science, Harvard Medical School, Boston MA, USA

${ }^{12}$ Fikst Product Development, Woburn MA, USA

${ }^{13}$ Department of Dermatology, Yale University School of Medicine, New Haven, CT USA 
${ }^{14}$ Department of Dermatology, Center for Cutaneous Oncology, Brigham and Women's Hospital; DanaFarber Cancer Institute, Boston, MA, USA

${ }^{15}$ System Design and Management, Massachusetts Institute of Technology, Cambridge, MA 02139, USA

${ }^{16}$ Massachusetts Manufacturing Emergency Response Team (MA M-ERT), Massachusetts Technology Collaborative, Westborough, MA 01581, USA

tThese authors contributed equally to this work

${ }^{*}$ Co-corresponding authors.

Correspondence:

peter_sorger@hms.harvard.edu cc: Maureen_Bergeron@hms.harvard.edu benle@mit.edu

‡Lead Contact: Peter K. Sorger

ORCID IDs:

Marc-Joseph Antonini, 0000-0002-9774-1483

Deborah Plana, 0000-0002-4218-1693

Shriya Srinivasan, $\mathrm{PhD}$, 0000-0002-2508-1324

Lyla Atta, 0000-0002-6113-0082

Aditya Achanta, 0000-0002-7610-3538

Avilash Kalpathy Cramer, 0000-0003-0014-8921

Jacob Freake, 0000-0002-5198-835X

Michael S. Sinha, MD, JD, MPH, 0000-0002-9165-8611

Sherry H. Yu, MD, 0000-0002-1432-9128

Nicole R. LeBoeuf, MD, MPH, 0000-0002-8264-834X

Peter Sorger, PhD, 0000-0002-3364-1838 


\begin{abstract}
The disruption of conventional manufacturing, supply, and distribution channels for medical supplies during the COVID-19 pandemic has caused widespread shortages and catalyzed local efforts to use nontraditional, rapid manufacturing to meet urgent healthcare needs. Here we present a crisisresponsive design framework designed to assist with product development under pandemic conditions. The framework utilizes extensive stakeholder engagement, comprehensive and dynamic needs assessment, local manufacturing, and product testing for the accelerated development of healthcare products. We contrast this framework with traditional medical device manufacturing and discuss relevant regulatory policies. We highlight the applicability of the crisis-responsive framework to a successful local program that designed and supplied face shields for a large US academic hospital. Finally, we make recommendations aimed at improving future resilience to healthcare emergencies. These include continued development of open source designs suitable for rapid manufacturing and changes in regulatory policy that strike a balance between rigidity and uncontrolled innovation.
\end{abstract}

Keywords: personal protective equipment (PPE); COVID-19; manufacturing; prototyping; 3D-printing; biocompatibility; sterilization; face shields; regulatory sciences; local resilience 


\section{INTRODUCTION}

\section{Rapid product development to meet emergent shortages of medical supplies}

In the face of a global COVID-19 pandemic, widespread disruption of international supply chains and distribution networks has led to severe shortages in personal protective equipment (PPE) and medical equipment such as ventilators ${ }^{1}$. These shortages have spurred numerous local efforts to supply alternative products. Many such efforts involve a diverse community of scientists, engineers, physicians, hobbyists (the "maker" community), community-based organizations and industrial manufacturers not previously involved in supplying healthcare products. Numerous international collaborations have been formed, anchored in open-source designs, rapid dissemination of pre-prints (on medRxiv or bioRxiv) and resources such as the National Institute of Health's 3D Print Exchange². Non-traditional fabrication of medical equipment is made feasible by the widespread availability and low cost of manufacturing techniques such as 3D printing and laser cutting. These approaches are ideal for low volume production of face shields, masks, frames for N95 filtering facepiece respirators ("N95 masks"), swabs for diagnostic kits, and potentially more complex medical products such as ventilator parts ${ }^{3,4}$. Local repair and rebuilding capacities have long been recognized as critical aspects of resilience to natural disasters and we propose that crisis-responsive local manufacturing be developed to the point it can be a key component of resilience to pandemics and healthcare emergencies at local and state levels ${ }^{5}$.

\section{A crisis-responsive design framework}

One of the greatest challenges facing non-traditional producers of medical equipment is the complex and unfamiliar regulatory landscape that is normally in place for these safety-critical products. Additionally, in many cases, design validation and testing are a bigger barrier than fabrication to the introduction of new or alternative products in an emergency setting. As a consequence, there have been multiple instances in which maker communities or small manufacturers have created a product in shortage only to find it turned away by healthcare providers and hospitals ${ }^{6}$. The primary goal of this 
paper is to prevent such situations by providing an overview of medical device development to makers, engineers, and manufacturers that are not traditionally involved in the medical industry. We also elaborate on development of design and regulatory frameworks relevant for future emergencies, with a focus on PPE and similar "low-risk" medical devices. Like the traditional framework for medical device development, a crisis-responsive framework incorporates systems-level interactions among producers and stakeholders that impact product development, testing and deployment. In a crisis however, it is necessary to reframe a traditionally deliberate, iterative, and highly controlled process for medical devices into a methodology that can be performed on an accelerated timescale without compromising product safety. Use of a crisis-responsive framework ensures that hospital incident commands, healthcare leadership, institutional review boards (ethics committees), product designers, and fabricators work efficiently together in pursuit of common goals.

Comparing traditional and crisis-responsive design frameworks for medical device development

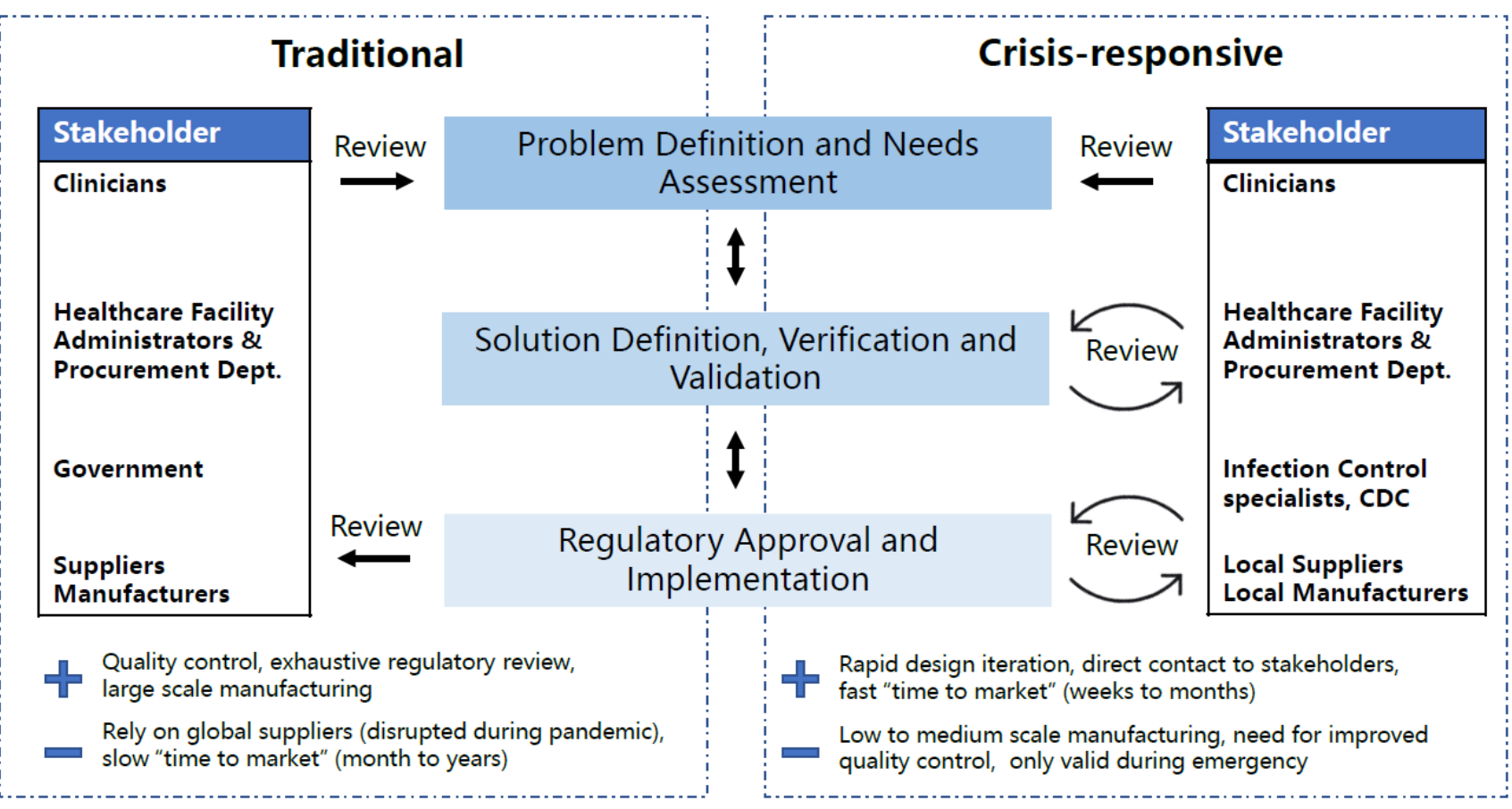




\section{Figure 1: Traditional and crisis-responsive design framework for medical device development.}

The crisis-responsive framework places emphasis on repeated design review using input from a broad range of stakeholders, all of whom may face unique constraints due to the pandemic. See also Figure S1.

A variety of models have been developed to describe medical device development. ${ }^{7-12}$ Key stages include: 1) problem definition and needs assessment, 2) solution definition, verification, and validation and 3) regulatory approval and implementation (Figure 1). Here we highlight two models: the traditional waterfall development process, first developed in 1970 to describe software development ${ }^{13}$ and historically used by most medical device manufacturers; and a crisis-responsive framework, better suited to tackle the rapidly-changing demands of the pandemic. This framework borrows from "agile product development" 14 and emphasizes flexibility, rapid implementation of new features to respond to changing requirements, and fast delivery of a working product. (Figure 1). A key difference between the two models is that waterfall development emphasizes feedback and iteration primarily at validation stages ${ }^{15}$, whereas crisis-responsive development involves review and iteration at earlier stages in a design; this is essential because it is rarely possible to formal market research or large-scale needs assessment under pandemic conditions. Use of agile product development and rapid manufacturing makes it possible to create finished prototypes on a time scale of days to weeks as opposed to months to years in traditional waterfall development, facilitating iterative design and testing with end-users. In a healthcare setting this is likely to include senior physicians and hospital leaders who would not normally be involved in PPE selection. Crisis-responsive development also relies on the willingness of hospital stakeholders to consider unfamiliar, innovative and costly designs based on an assessment of risks posed by the unavailability of traditional products.

In conventional needs assessment, the impact of selling price, access to retail and wholesale channels and branding are carefully considered. For commodity products lacking strong intellectual property protection (e.g. face shields, N95 masks, gowns), selling price may be the strongest limitation on design and quality. For proprietary products, the willingness of third parties or national healthcare 
systems to pay for the product is important. Price sensitivity also varies among private and public health systems, nursing homes, and independent living facilities, leading to a plethora of functionally similar products with different features. In a crisis, however, many of these concerns are no longer as relevant since the goal is typically to produce the best possible product in the shortest amount of time, given limitations in fabrication facilities, materials, and the skill of the design team; price, branding and channel access are of minimal importance. These changes fundamentally alter the stakeholder landscape and the design process.

The crisis-responsive framework has many potential limitations in terms of quality and sustainability and is not suitable for use under non-emergency conditions; it is intended to provide rapid-turn stopgap solutions to meet immediate needs. Crisis-responsive design typically does not include the documentation needed for regulatory review. It is instead often based on small-scale production and sensitive to unanticipated substitution of input materials due to supply shortages, and it does not involve brand identity or thorough analysis of intellectual property. Despite these limitations, even in a crisis it is essential that a rational and well-considered process be followed to ensure that products are as functional, reliable and safe as possible. Only then can manufacturing by local communities effectively respond to urgent shortages in medical devices.

\section{Stage 1: Problem Definition and Needs Assessment}

The importance of stakeholder input

Traditionally, problem definition involves assessing the needs of end-users through market research. In the case of PPE this is articulated by healthcare systems not individuals. However in a crisis, it is common to work directly with end-users for PPE procurement, including healthcare workers caring for sick patients. These individuals will be most concerned with the usability, reliability, and testing of a product. Design, manufacturing, distribution, risk-mitigation and lifecycle considerations (e.g. sterilization) remain the purview of the design and fabrication team, but we have found that end- 
users are often remarkably knowledgeable about and willing to engage in issues of design and fabrication: do not overlook their contributions in this area.

The process of engaging stakeholders will differ in private and public hospitals, private practices, nursing homes, and independent living facilities. Input from administrators and purchasing or procurement departments is important to better understand current needs. Local and state government officials can be a resource for regulatory, purchasing power, and supply chain information and should be consulted if possible. In many cases, non-traditional medical products use components that were manufactured for other purposes (e.g., HEPA vacuum cleaner filters for use in PAPRs). Local suppliers and distributors can be an invaluable source of information on the availability of such materials and equipment and their technical performances; we have found that, during the COVID-19 pandemic, many materials suppliers are willing to provide extra help to fabricators who are not part of their traditional customer base. A final and important aspect of needs assessment is incorporating the needs of a diversity of end-users who differ in gender, body size and shape (e.g. differing face dimensions in the context of respirators) or in clinical role (e.g. nursing staff vs. physicians in emergency rooms vs. outpatient consultation).

\section{Coordinating with multiple stakeholders}

At the outset of the COVID pandemic, many municipalities and even hospitals had their own design and fabrication teams working largely independently of each other, although often using shared designs and methods. Several months into the pandemic, particularly after the first wave of hospitalization passed, local fabrication teams started to work together to improve efficiency and share expertise. State programs such as Massachusetts Manufacturing Emergency Response Team (M-ERT; https://masstech.org/M-ERT) and national efforts such as America Makes (https://americamakes.us/) are playing an increasingly important role in matching end-users with suppliers and providing access to tested designs, materials and supply chains. Including such groups in the design and fabrication 
process can bring substantial benefits in terms of the suitability of the design and feasibility of fabrication.

\section{Traditional and nontraditional supply procurement}

The first stage in meeting urgent supply shortages is to look for alternative medical manufacturers of similar products. When such products are not available, an alternative solution is to find non-medical suppliers of functionally related products and components. For instance, the Greater Boston Pandemic Fabrication Team (PanFab) ${ }^{16}$ PAPR design uses commercially available vacuum filters since supply shortages made it challenging to procure filters traditionally used in healthcare settings ${ }^{16}$. In addition to meeting device shortages through nontraditional manufacturing of existing products, the design of entirely new products can help meet PPE shortages in novel ways. For instance, many N95 respirators become unusable after several donning and doffing cycles due to poor fit of the nosepiece (the metal tabs often become distorted) and degradation or breakage of the elastic straps that hold masks in place. Because N95-type respirators require highly specialized fabrics and equipment to manufacture, it is more feasible to fix the problems with existing masks than to make new ones de-novo. As a result, multiple groups have developed 3D-printing mask frames that can fit over existing N95 masks and take the place of degraded or broken nosepieces and straps. ${ }^{17-23}$ Like many other innovative products developed to meet emergency needs, mask frames may also have role in respiratory protection under non-crisis conditions.

\section{Determining raw materials needs}

A needs assessment in a crisis must not only consider the needs of the end-users, but also the capabilities of manufacturers and suppliers. During a pandemic, acquiring raw materials may be particularly challenging, as suppliers may be closed or an entire class of material may be out of stock (e.g. thin BoPET sheets commonly used in face shields). It is therefore important to consider equipment and supply constraints early in the design process and to assess alternatives, such as raw material 
substitutions and alternative fabricators or suppliers. In a crisis, multiple fabricators can collaborate to increase production volume and provide complementary capabilities.

Converting needs assessment results to technical specifications

Requirements identified via needs assessment must be converted into functional and technical specifications that guide design. For example, if an end-user needs a face shield that is adaptable to different individuals, the functional requirement includes ensuring coverage of different size faces and making straps and attachment hardware adjustable. The technical specification might then be a shield of length of 22.5 to $30 \mathrm{~cm}$ and headband circumference of $50-60 \mathrm{~cm}$. Analogously, an end-user requirement for reusability triggers a requirement for input from infection control experts and results in a functional specification for designs and materials that can be sterilized. The technical specification would then call for materials compatible with sanitizing wipes or hydrogen peroxide sterilization and an absence of crevices that can trap contaminants.

\section{Stage 2: Solution Definition, Verification and Validation}

Solution definition, verification and validation is an iterative process in which functional and technical specifications defined in stage 1 are transformed into actual designs. Designs are then compared to specifications to verify that all requirements have been met. General considerations applicable to all medical products must also be included, such as the biocompatibility of materials in contact with humans. During a crisis, very high demand for some types of equipment and materials may impose additional requirements on components, materials and processes.

\section{Rapid Manufacturing Techniques}

Rapid manufacturing methods have a well-established role in facilitating rapid cycles of design and testing to reduce uncertainty and shorten production timelines. ${ }^{24}$ The use of rapid manufacturing methods is increasing in healthcare, facilitated in part by a series of FDA workshops that resulted in the 
2017 publication of "Technical consideration for additive manufactured medical devices"25. In the case of prosthetics ${ }^{26}$ and orthotics ${ }^{27}$, tools for surgical planning ${ }^{28}$, and dental and surgical equipment ${ }^{27,29,30}$ additive manufacturing has opened new possibilities for designing products with complex geometries and allowed manufacturers to move away from standardized products in a few pre-specified sizes toward patient-matched products. During the COVID-19 pandemic, additive manufacturing has been widely used to make face shields, ${ }^{27,31,32}$ nasopharyngeal swabs ${ }^{33-35}$, face mask brackets ${ }^{18}$, components for portable-air purifying respirators (PAPRs) $)^{35}$, and ventilator splitters ${ }^{36}$. In response, the FDA has released relevant guidance in its " $F A Q s$ for $3 D$ printing medical devices, accessories, and components during the COVID-19 pandemic." ${ }^{3}$ Box 1 outlines key medical supplies currently in shortage, and the manufacturing modality/modalities that could be used to produce their alternatives.

Rapid manufacturing is infrequently used with PPE under normal circumstances; such methods are typically more expensive (per unit) than conventional large-scale production (e.g. injection molding). The limited production volumes of rapid manufacturing also pose a significant challenge to meeting the large demand created by the pandemic. Thus, they are most effective as a means for prototyping and short-term production while large-scale production methods ramp up. 
Box 1. Prototyping and manufacturing methods applicable to production of five medical devices, based on Open Source COVID-19 Medical Supply Guide. ${ }^{37}$

\begin{tabular}{|c|c|c|c|c|c|c|c|c|c|}
\hline Categories & $\begin{array}{c}\text { Set-up Cost } \\
\text { and Time }\end{array}$ & $\begin{array}{l}\text { Prod. Cost } \\
\text { and Time }\end{array}$ & Specific Methods & $\begin{array}{l}\text { Face } 1 \\
\text { Shields }\end{array}$ & $\begin{array}{l}\text { Nasapharyngeal } \\
\text { Swabs }\end{array}$ & $\begin{array}{l}\text { Surgical } \\
\text { Face Masks }\end{array}$ & $\begin{array}{c}\mathrm{N} 95 \\
\text { Respirators }\end{array}$ & PAPRs & $\begin{array}{c}\text { Ventilator } \\
\text { Splitters }\end{array}$ \\
\hline \multirow{4}{*}{$\begin{array}{c}\text { Rapid } \\
\text { Prototyping }\end{array}$} & \multirow{4}{*}{ Low } & \multirow{4}{*}{ High } & 3D Printing (FDM) & Yes & Yes & No & Yes & Yes & Yes \\
\hline & & & 3D Printing (SLA) & Yes & Yes & No & Yes & Yes & Yes \\
\hline & & & Machining & Yes & No & Yes & Yes & Yes & TBD \\
\hline & & & Laser Cutting & Yes & No & Yes & No & Yes & No \\
\hline \multirow{4}{*}{$\begin{array}{l}\text { High Volume } \\
\text { Production } \\
\text { Processes }\end{array}$} & \multirow{4}{*}{ High } & \multirow{4}{*}{ Low } & Die Cutting & Yes & No & Yes & Yes & Yes & No \\
\hline & & & Injection Molding & Yes & Yes & No & Yes & TBD & Yes \\
\hline & & & Compression Molding & Yes & Yes & No & No & TBD & TBD \\
\hline & & & Thermoforming & Yes & Yes & No & No & TBD & TBD \\
\hline \multirow{5}{*}{$\begin{array}{l}\text { Fabrication } \\
\text { and } \\
\text { Assembly }\end{array}$} & \multirow{5}{*}{ Variable } & \multirow{5}{*}{ Variable } & Sewing & No & No & Yes & Yes & Yes & No \\
\hline & & & Gluing and Bonding & Yes & Yes & Yes & Yes & Yes & TBD \\
\hline & & & Fastening & No & No & No & No & Yes & No \\
\hline & & & Electronics & No & No & No & No & Yes & No \\
\hline & & & Assembly & Yes & No & Yes & Yes & TBD & Yes \\
\hline
\end{tabular}

\section{Definitions:}

FDM: Fused Deposition Modeling. An additive manufacturing (3D printing) process that can be implemented using low-cost consumer-grade equipment. A printer heats up a plastic in filament form and uses a print head to create a 3D structure from the bottom up.

SLA: Stereolithography. Another additive manufacturing process using low cost consumer-grade equipment in which a vat of liquid resin is hardened with a laser to create desired shapes.

Machining: Conventional (subtractive) manufacturing in which raw material is cut into a desired shape by a controlled material-removal process such as milling and routing. Small, low-volume, computer numerical control (CNC) machines are increasingly capable and widespread but their throughput is limited.

Laser cutting: A technology in which a high-powered laser is used to cut sheet materials to size; consumer-grade laser cutters for plastics are inexpensive although limited in accuracy and throughput. 
Die cutting: A technology in which metal knives (dies) with sharp edges are pressed into sheet materials to cut it to size and shape. Production time per piece is significantly faster than laser cutting but requires more expensive and longer setup due to the custom-fabrication of the die.

Waterjet cutting. A technology in which a narrow jet of high pressure water and abrasive is used to cut sheets of material; desk-top waterjet machinery is now available.

Injection Molding: A high-volume manufacturing technology that produces parts by injecting molten material (polymers, glass, metal) into a mold. The upfront cost and setup time of injection molding is high but unit cost and production time is low.

Compression molding: A system by which heat and pressure are applied to a material in order to shape it.

Thermoforming: A technique by which plastic is heated, formed to a specific shape in a mold, and trimmed to create a desired shape.

\section{Sterilization and reuse}

To address acute shortages in traditionally single-use devices such as respirators or face shields, the $C D C$ has issued guidance allowing extended-use, reprocessing, and reuse of $P^{2} E^{38}$. According to FDA regulations, hospitals and third-party reprocessors are considered "manufacturers" of the reprocessed devices and must comply with the same regulatory requirements as the original equipment manufacturers ${ }^{39}$. While FDA and CDC guidelines have been relaxed for single-use devices, designers should take the necessary steps to ensure compatibility with sterilization, decontamination and cleaning procedures. Additionally, fabricators should take the appropriate steps to ensure initial disinfection and sterilization of their products prior delivery to institutions. The EPA's "list $\underline{\mathrm{N}}$ :Disinfectants for Use Against SARS-CoV-2" provides a detailed record of products meeting EPA's criteria for use against SARS-CoV-2, along with corresponding directions for use $^{40}$.

In a pandemic, many products that are normally disposable end up being reused because they are in short supply. It should therefore be assumed that face shields, masks frames and other items will 
be sterilized or decontaminated if at all possible. The common sterilization methods of autoclaving, bleach, and alcohol-based wipes are well-suited for the initial sterilization of products such as face shields. However, these methods often degrade labels and safety warnings and they are not compatible with products such as N95 masks. Short wavelength ultraviolet (UV) light ${ }^{41}$, vaporized or ionized hydrogen peroxide ${ }^{42-44}$, and moist heat are alternative possibilities often available in healthcare settings ${ }^{41}$. Determining the compatibility of rapidly manufactured products with available sterilization methods should be done early into the design process. The use environment should also be taken into consideration; for example, access to the sterilization equipment or decontamination solutions may be limited during a crisis and the process of getting products to and from centralized sterilization facilities must be considered. In many cases, the fundamental desirability of product reuse runs up against practical challenges with logistics. This is particularly true in the case of PPE that needs to be sterilized or decontaminated and then returned to the original users. We have found that many healthcare providers are not willing to put the necessary tracking procedures in place to make "return to original user" possible.

\section{Biocompatibility}

Biocompatibility is defined by the FDA as the "ability of a device material to perform with an appropriate host response in a specific situation" 45 where response refers to a host immune or inflammatory reaction to the material. Evaluation of biocompatibility is one part of the FDA's overall determination of safety and effectiveness for new or modified devices that come into direct or indirect contact with the human body ${ }^{46}$. In the US, two documents outline standard biocompatibility testing: the International Standard ISO 10993-1, "Biological evaluation of medical devices - Part 1: Evaluation and testing within a risk management process" 47 and the guidance related to ISO 10993-145. A separate biocompatibility standard exists specifically for respiratory devices: ISO 18562-1:2017, "Biocompatibility evaluation of breathing gas pathways in healthcare applications." ${ }^{48}$ Among other factors, a biocompatibility assessment focuses on: (1) material chemistry and any changes caused by the 
manufacturing process, (2) material physical properties, (3) nature of the body contact (direct or indirect), (4) contact duration, and (5) prior history of safe use (as defined in ISO 10993-145).

Biocompatibility requirements for materials are application-specific and vary greatly based on the part of the body in contact with the device and the duration of the contact; sustained internal contact is substantially more problematic than brief or external contact with the skin. Thus, approval of a material in one application does not constitute approval for another application. In addition to material considerations, the method by which a material is handled or processed during manufacturing may influence its biocompatibility. During biocompatibility evaluation, testing is performed on the "final finished form" of the device, which includes all manufacturing processes including packaging and sterilization. Rapid prototyping can be advantageous in conducting biocompatibility testing early in a product development cycle.

From a practical standpoint, most devices being subjected to rapid fabrication for pandemic response are for external use only and primarily contact the skin (or hair). In this setting, it is reasonable to use materials previously shown to be safe, such as silicones, parylene, and common fabrics. Particular attention should be paid to foam, elastic materials and adhesives with respect to skin contact and latex should be avoided; Monprene ${ }^{\circ}(\mathrm{PR}-23040)$ is an FDA-approved alternative that is widely used in phlebotomy and readily available.

If materials previously documented to be biocompatible in a particular setting are unavailable or functionally unsuitable, then biological endpoints ranging from cytotoxicity and sensitization to material degradation and carcinogenicity must be considered. Attachment A of FDA's guidance related to ISO 10993-1 provides a list of the recommended biological endpoints to consider for the development of biocompatibility evaluation as well as the rationale for these endpoints ${ }^{45}$. The flow chart of Figure 2 illustrates how one might evaluate biocompatibility to determine if a newly-developed device requires supplementary testing. During the current crisis, safeguards have been relaxed by regulatory authorities to enable more rapid response, so long as a specific medical device's product code is explicitly mentioned in an FDA guidance or enforcement policy. These guidance documents are freely 
available on the FDA website (https://www.fda.gov/emergency-preparedness-and-

response/coronavirus-disease-2019-covid-19/covid-19-related-guidance-documents-industry-fda-staffand-other-stakeholders).

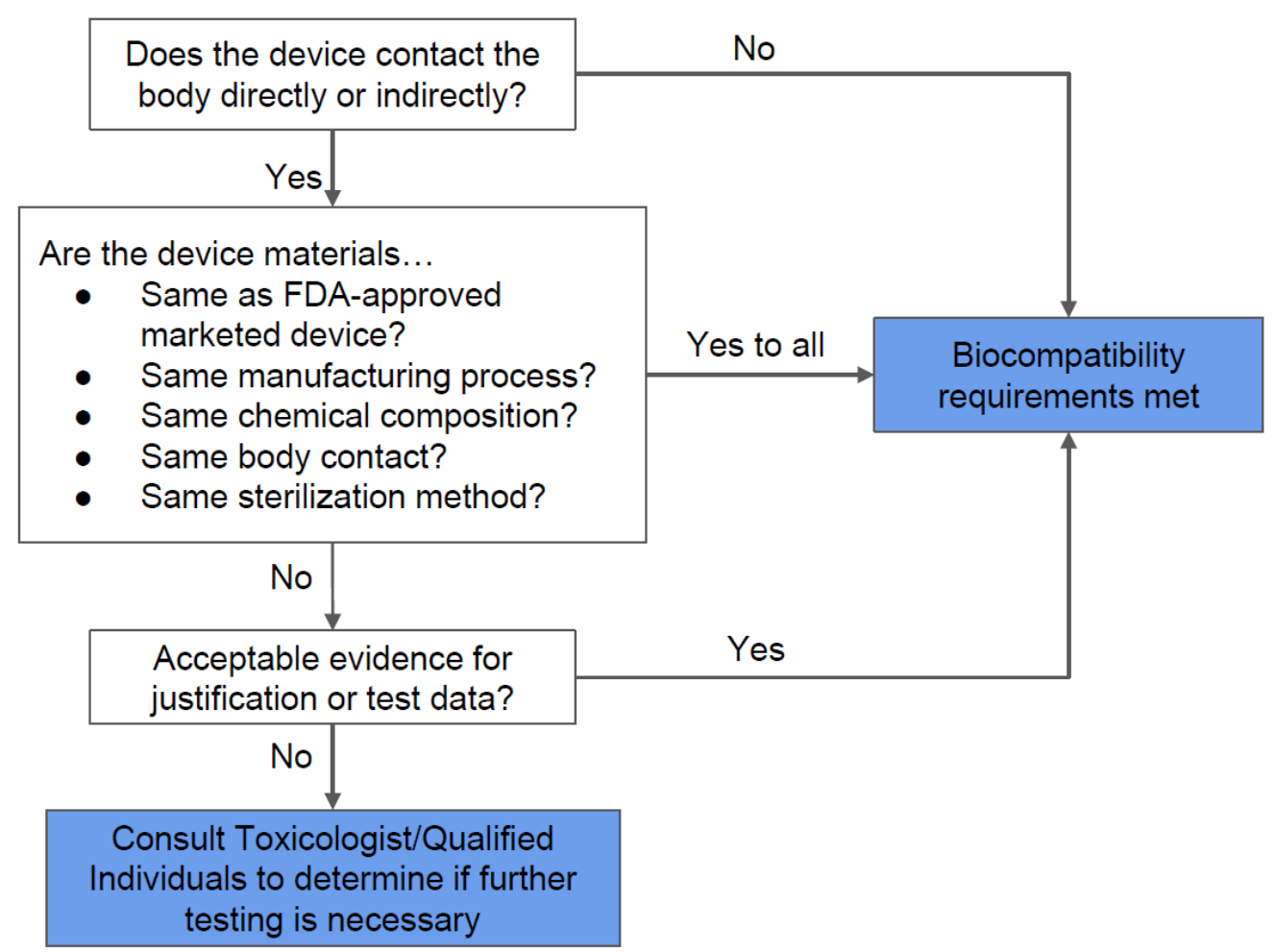

Figure 2: Systematic approach to a biological evaluation of medical devices as part of a risk management process. The process is simplified from FDA Guidance document for ISO 10993-145.

\section{Design Verification and Validation (V\&V)}

Design verification is an iterative and empirical process in which objective evidence is sought to assess whether a product satisfies specifications. Design validation is a summative exercise that assesses the integrity of the final product and ensures that it meets user needs in a real or simulated use environment via testing, measurement, and observation of user interaction (commonly to ISO 13485:201649). It should be noted that a product's packaging, labeling, and instructions are considered to be essential parts of the product. Even in a crisis it is important to provide inserts and labels with 
products describing their intended use, composition, and regulatory compliance (e.g. reference to an EUA).

Table 1: Summary of regulatory and testing standard for each device

\begin{tabular}{|c|c|c|c|c|}
\hline $\begin{array}{l}\text { Medical } \\
\text { supply }\end{array}$ & $\begin{array}{l}\text { Device } \\
\text { FDA } \\
\text { Product } \\
\text { code }\end{array}$ & $\begin{array}{l}\text { Traditional } \\
\text { Regulatory } \\
\text { Process }\end{array}$ & $\begin{array}{l}\text { Revised process during COVID-19 } \\
\text { pandemic }\end{array}$ & $\begin{array}{l}\text { Standards for testing } \\
\text { (NIOSH/ANSI) }\end{array}$ \\
\hline $\begin{array}{l}\text { Face } \\
\text { shield }\end{array}$ & LYU & $\begin{array}{l}\text { FDA } \\
\text { regulated } \\
\text { Class I } \\
510(k) \\
\text { exempt }\end{array}$ & $\begin{array}{l}\text { FDA Emergency Use Authorization } \\
(\text { EUA })^{54} \\
\text { FDA Enforcement Policy }\end{array}$ & ANSI/ASSE Z88.2-2015 \\
\hline $\begin{array}{l}\text { Nasophar } \\
\text { yngeal } \\
\text { (NP) } \\
\text { swabs }\end{array}$ & $\begin{array}{l}\text { KXF } \\
\text { KXG }\end{array}$ & $\begin{array}{l}\text { FDA } \\
\text { regulated } \\
\text { Class I } \\
510(k) \\
\text { exempt }\end{array}$ & No EUA & $\begin{array}{l}\text { Not applicable. See text } \\
\text { for description of } \\
\text { materials, PCR } \\
\text { compatibility, transport } \\
\text { media compatibility, } \\
\text { mechanical performance, } \\
\text { and length } \\
\text { considerations. }\end{array}$ \\
\hline $\begin{array}{l}\text { Surgical } \\
\text { face } \\
\text { masks }\end{array}$ & FXX & $\begin{array}{l}\text { FDA } \\
\text { regulated } \\
\text { Class II } \\
510(k)^{81}\end{array}$ & FDA Enforcement Policy ${ }^{82}$ & $\begin{array}{l}\text { 6-254 ASTM F2100-11; } \\
\text { 6-335 ASTM F2101-14; } \\
\text { 6-406 ASTM F1862; 6- } \\
\text { 425 ASTM F2100-19; 6- } \\
\text { 427 ASTM F2101-19 }\end{array}$ \\
\hline $\begin{array}{l}\text { N95 } \\
\text { respirator }\end{array}$ & $\begin{array}{l}\text { ONT, } \\
\text { ORW, } \\
\text { NZJ }\end{array}$ & $\begin{array}{l}\text { FDA } \\
\text { regulated } \\
\text { Class II } \\
510(\mathrm{k}) \\
\text { clearance }\end{array}$ & $\begin{array}{l}\text { FDA Emergency Use Authorization } \\
(\text { EUA })^{55}\end{array}$ & $\mathrm{NIOSH}^{83,84}$ \\
\hline PAPR & N/A & $\begin{array}{l}\text { NIOSH } \\
\text { regulated, } \\
\text { FDA } \\
\text { approved }^{85}\end{array}$ & $\begin{array}{l}\text { FDA Emergency Use Authorization } \\
(\text { EUA })^{55} \text { as a subset of filtering } \\
\text { facepiece respirators (FFRs) }^{86}\end{array}$ & $\mathrm{NIOSH}{ }^{83}$ \\
\hline
\end{tabular}

${ }^{*}$ Only true for the duration of the crisis, not applicable after EUA is no longer enforced. These EUA guidelines are up to date as of the time of submission 


\section{Scaling up}

While rapid manufacturing techniques such as 3D printing and laser cutting are efficient approaches for fabricating prototypes, low throughput and high unit costs do not make them feasible for large-scale production. In a traditional waterfall process, once a design process converges on a final set of specifications, design transfer takes place during which the design is adapted to the demands of large-scale manufacturing methods such as injection molding and die cutting. These methods are highthroughput and inexpensive per piece but are associated with significant cost and set-up time (up to several months). Moreover, highly specific expertise is generally required to make design dies and molds compatible with a specific type of equipment. In a crisis, in which time is usually limited and production costs are less of a concern, parallel production using the prototyping facilities of many manufacturers, colleges, and makers can sometimes meet demand. For example, in the case of the Panfab/BWH face shield (Box 2), 3000 face shields were fabricated in a few weeks using 3D printing and laser cutting at multiple sites ${ }^{32}$ and the Czech 3D producers PRUSA described a highly parallel face shield printing process using inexpensive machines ${ }^{50}$.

Conversations with suppliers and manufacturers will help guide prototyping and design processes and prepare for design transfer to a manufacturer, if relevant. A responsible entity may be required to register with the FDA as a manufacturer and list the product(s) they are distributing or selling, or the services they are providing to other manufacturers, depending on the product code, respective risk classification, and regulatory compliance pathway ${ }^{51}$. Meeting these requirements will be especially important once the public health emergency has ended. Additional stakeholders such as payers and post market surveillance organizations may also come into play (Supplementary Figure S1).

\section{Stage 3: Regulatory approval and Implementation}

On January 31, 2020, the US HHS Secretary Alex Azar declared a public health emergency involving COVID-19, noting that the circumstances justified emergency use of in vitro diagnostics and 
other medical devices that aid in the detection or diagnosis of COVID-1952. Pursuant to this declaration, the FDA has issued Emergency Use Authorizations (EUAs) for a number of medical devices, with the specific code for each product stated in the document. As mentioned above, EUAs allow certain nonFDA approved medical products to be used in the absence of adequate FDA-approved alternatives ${ }^{53}$. While some EUAs are manufacturer-specific, others are broader in scope. For example, EUAs for face shields and respirators waive certain FDA requirements for all prospective manufacturers, authorize the modification of approved products, allow for extended use, or allow for widespread production and distribution of devices so long as manufacturers adhere to requirements outlined in the EUA notice ${ }^{54,55}$. EUAs expire upon resolution of the public health emergency.

The agency has also provided extensive and frequently updated guidance documents for manufacturers seeking to produce rapid diagnostics, personal protective equipment (PPE), and other devices for front-line use in responding to COVID-19. EUAs have also extended into the realm of sterilizing PPE for re-use.

A lack of regulatory oversight does not absolve designers and fabricators from doing their best to ensure that products are safe and functional and do not put healthcare providers or patients at risk. Additionally, disclosure of risk to the end-user is important: the FDA EUAs and enforcement policies include specific requirements for labeling, information for use, and use environments to ensure that products do not create undue risk.

\section{Overview of Medical Device Regulatory Review \& Implementation}

In the US, medical devices are typically made available via the FDA's 510(k) premarket notification process. Depending on the degree of risk associated with the use of a medical device it is classified by the FDA as either Class I (low risk), Class II (moderate risk), or Class III (high-risk) ${ }^{56,57}$. The 510(k) submission process requires that a manufacturer of a device new to the market demonstrate "substantial equivalence" to one or more legally marketed devices, thereby avoiding a requirement for extensive clinical testing. Substantial equivalence does not require that a device be 
identical, but instead mandates that it be as safe and effective as an existing marketed device ${ }^{58}$. Some Class I products are exempt from the 510k premarket submission process based on the evaluation that these products pose a particularly low risk. This includes devices such as face shields, most forms of PPE, and nasopharyngeal swabs. Class II devices include more complex live-critical products such as ventilators and Class III devices traditionally encompass those implanted into a person, such as pacemakers, defibrillators, or prosthetics. Almost all devices currently being supplied by non-traditional fabricators and maker communities fall into Class I, and the majority are Class I exempt. However, ventilator splitters, which have been much discussed as a way of increasing ventilator capacity ${ }^{59}$, may be Class II devices ${ }^{60}$ and considerable controversy has attended their development ${ }^{61}$.

The premarket submission process only clears a manufacturer to market a product; additional requirements must be met prior to manufacturing, selling, or distributing it. Quality controls in manufacturing, referred to as good manufacturing practices (GMP), include a requirement of product traceability in case of manufacturing flaws or product recalls. Notably, no Class II devices, and only a small number of Class I 510k exempt devices, are also exempt from GMP regulations ${ }^{62}$. Meeting GMP standards constitutes a substantial barrier for formal certification of devices fabricated through local, nontraditional manufacturing practices.

After implementation, unique device identification, an important part of the FDA's post-market surveillance process, ensures that manufacturers vigilantly and proactively monitor the use of their product(s) for adverse events and patient injuries. The post-market surveillance process can also act as means to collect user feedback, which can lead to product improvements or innovative alternatives to existing designs. In the current crisis, US regulators have put in place emergency authorizations that waive some of the normal GMP and tracing requirements in favor of less stringent discretionary enforcement. In many cases, this forms the regulatory foundation for repurposing products from the non-medical supply chain for and for hospitals to engage with informal networks of maker communities and similar organizations ${ }^{63}$. The underlying logic is an attempt to balance the risks associated with a lack of supply against the risks of using new designs and non-traditional manufacturing processes 


\section{Product safety validation for PPE}

Product safety validation for PPE and similar low-risk devices is commonly performed by certified testing laboratories, many of which are commercial and provide fee-based testing for compliance with specific regulatory standards (e.g. NIOSH standards for PPE). Under normal circumstances, regulations require that quality management systems (QMS, e.g. to ISO 9001) be in place; a QMS specifies how design and development inputs and outputs should be recorded, records maintained on the skills, experience and qualifications of key personnel, and calibration records established and monitored. Statistical Process Control (SPC) is also widely used throughout medical device manufacturing to ensure product consistency and quality. In a crisis-responsive setting, QMS and SPC are likely to be infeasible to put in place quality management systems may be infeasible, which makes formal certification to NIOSH or FDA standards impossible. In such instances, makers must do their best to maintain quality and test either independently or via the use of other local resources such as academic laboratories. Consortia like the Mass General Brigham Center for COVID Innovation ${ }^{64}$, N95Decon ${ }^{65}$, and M-ERT66 may be able to provide guidance in specific situations. Commercial laboratories can also perform specific aspects of device testing, even if true certification is not possible.

\section{Use of research protocols}

One way to test non-traditional medical products in a healthcare setting is to use research protocols overseen by an Institutional Review Board (ethical review board; IRB); these are part of the normal operation of virtually all teaching hospitals. The use of a research protocol makes clear to participants, via the process of informed consent, that a product is being tested and that it has not necessarily been through the usual regulatory review. IRB approval is also almost always required for conducting user surveys and receiving direct feedback from end-users. Devices such as face shields 
(described in Table 1) are FDA regulated Class I products with the lowest risk to human health and are therefore exempt from many regulatory requirements. They are therefore suitable for testing in an IRBsupervised research protocol under even the suboptimal conditions of a healthcare emergency. We have found that the use of research protocols for product testing increases buy-in from healthcare leadership, in part because it is a familiar process. In the case of a non-traditional face shield described below (Box 2) it was possible to perform IRB protocol review and product specification, validation, and testing in a clinical setting in a period of roughly three weeks ${ }^{32}$.

\section{A case on fabricating a face shield in the northeast U.S.}

In response to the COVID-19 pandemic, which grew rapidly in Massachusetts during March 2020 , multiple teams formed to address rapidly growing shortages in medical supplies. Some of these teams were established by institutional mandate and others arose spontaneously through the efforts of engaged individuals. An example of the latter is the Greater Boston Pandemic Fabrication Team (PanFab; https://www.panfab.org/), a student-faculty initiative organized by the Harvard-MIT Center for Regulatory Sciences. It consists of a group of volunteers with expertise in engineering, biomedicine, manufacturing and regulatory review working closely with the physicians at Boston-area hospitals, the local maker community and manufacturing experts from local companies contributing outside of normal work hours. A physician assigned to a hospital incident command was a particularly important member of the PanFab team because she could provide timely and accurate information on current and emerging needs. PanFab has been effective in designing and rapid fabricating face shields, mask frames, PAPRs and other types of PPE ${ }^{32}$ and its activities are representative of local design in response to a healthcare crisis. 


\section{Box 2. Case study: nontraditional design and fabrication of face shields during a health care emergency.}

\section{Stage 1: Problem Definition and Needs Assessment}

Defining the problem:

Face are a critical component of PPE per the $\mathrm{CDC}^{67}$ and are used in conjunction with surgical masks or N95 FFRs to protect the face and neck, particularly mucous membranes in the eyes, nose, and mouth, from splatter by contaminated bodily fluids ${ }^{68}$. However, during the COVID-19 pandemic, a severe shortage of face shields developed. Face shields are composed of a clear shield (commonly made from BoPET, PETG, acetate, or polycarbonate) and headband frame that is in contact with a user's forehead and commonly made from a lightweight plastic or foam ${ }^{68}$. In a US health care setting, face shields are traditionally single use devices, but because of short shortages they are being worn for the full duration of a shift ( 6 to 12 hours) and then being sterilized for reuse.

Needs assessment results and associated technical specifications:

From interviews with healthcare providers, hospital administrators, and infection control specialists, the following needs were identified:

1) The shield must protect mucosal membranes from splashes of bodily fluid by extending from the forehead to the points of both ears and down the neck.

1) The shield should not fog or otherwise obscure the user's view, even during strenuous activity that may produce perspiration ${ }^{68}$. 
2) The shield should fit a range of facial lengths and heights while not interfering with range of head and neck motion.

a) Associated Technical specification: Facial lengths $23-30 \mathrm{~cm}$ and range of motion 180 degrees in each direction

3) The shield should remain firmly in place and remain comfortable when the user moves their head up, down, and laterally at varying speeds

4) Face shields are typically worn for the duration of a shift (up to 12 hours). Thus, the shield cannot be so heavy as to cause discomfort when worn for a shift.

5) Attachment mechanisms should remain firm while preventing skin sensitization, irritation, or imprints on the skin. The attachment must be adjustable.

a) The FDA EUA allows specific materials to be re-used without thorough re-testing; these are materials of choice for the design.

6) Device cost should be less than $\$ 5$ per shield

7) Hospital demand requires a production rate of 2000 shields per month

8) The face shield should require minimal assembly, both for ease of use and to limit the presence of hard to reach surfaces, which could interfere with the decontamination process

9) To be reusable, the face shield should be compatible with the hospital's commonly used sterilization techniques, which include ionized hydrogen peroxide, germicidal disposable wipes, or $70 \%$ isopropanol wipes.

\section{Stage 2: Solution Definition and Validation}

\section{General Recommendations}

A crisis-responsive design framework aims to couple user needs, rapid manufacturing technologies and local fabrication to fulfill unmet demand for simple medical devices in times of crisis. The following recommendations are intended to serve as a checklist for designers, maker communities 


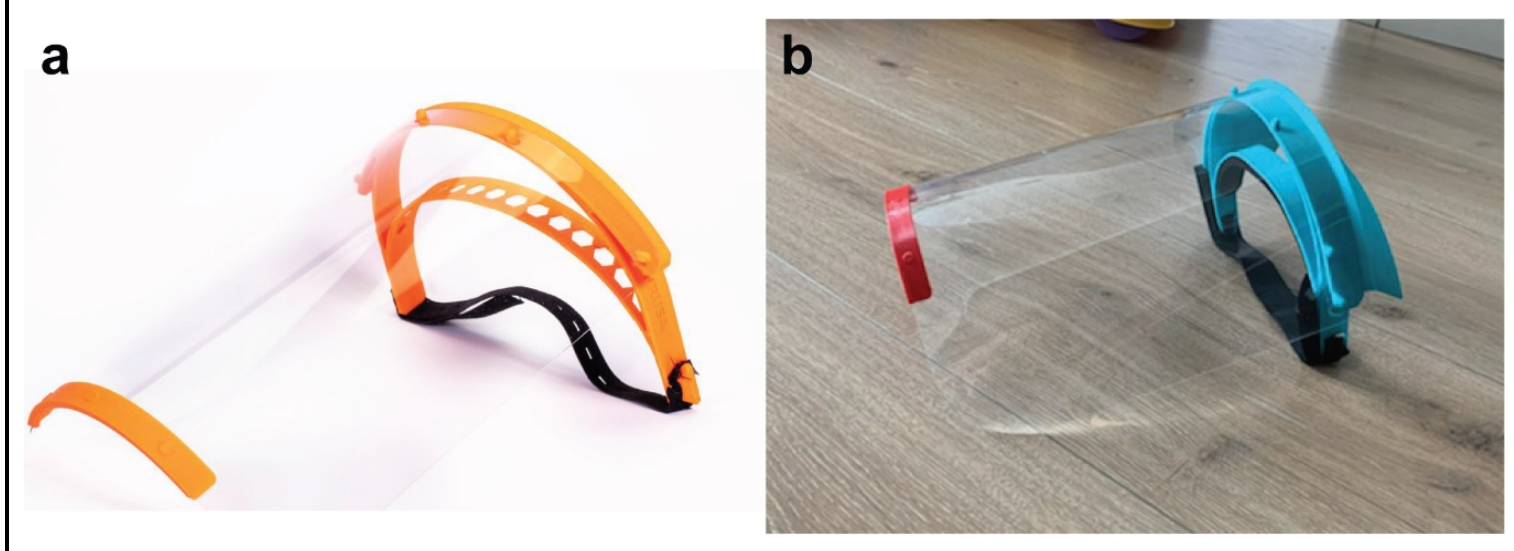

Figure 3: Example face shields fabricated during the COVID-19 pandemic. A) Image of Prusa RC2 design and B) final PanFab face shield prototype. See text for references and details.

\section{Prototyping and Manufacturability:}

Given the low-risk nature of the device, various 3D printing approaches were considered acceptable. We began with the open-source Prusa design ${ }^{31}$ and iterated it based on feedback from healthcare providers at Brigham and Women's Hospital (Figure 3). The new design added a forehead fin with a drip guard that protected the otherwise-exposed forehead from body fluid exposure. This was requirement was not identified during initial assessment but became obvious once prototypes were in the hands of emergency room users. The prototype shield was too narrow and short and did not provide sufficient splash protection for the neck and sides of the face for all users; the length and width of the shield was therefore increased.

\section{Sterilization and reprocessing:}

Given that safe sterilization of 3D printed face shields had not been extensively studied, we followed CDC's guidance ${ }^{69}$ relating to sterilization and reprocessing compatibility for googles, a related product. We checked for changes in material properties and visor transparency following several days of use and following regular cleaning with sanitizing wipes (Germicidal Disposable Wipes). We also ensured that the face shield could withstand ionized hydrogen peroxide 
sterilization (iHP; TOMI SteraMist), resulting in effective killing of test bacterial spores as measured by standard biological indicators. ${ }^{70}$

\section{Biocompatibility}

A number of materials were assessed during fabrication of the face shield based on (i) resource availability, (ii) previous uses in marketed and FDA-approved medical devices, and (iii) compatibility with common sterilization techniques. In the case of the face shield, only the 3D printed headband, the foam pad and the Velcro strap of the face shield were in contact with user's skin or hair.

Applying the workflow shown in Figure 2, the requirement for safe interaction with intact skin was met based on limited duration body contact $(<24 \mathrm{hrs})$ and prior material biocompatibility information obtained from material MSDS, reported experience from manufacturers, and published literature . PLA was selected as the material for 3D printing the headband because of its wide availability and known safety in contact with the skin. The Velcro strap, which is made from polyethylene and nylon, is also known to be safe for skin contact. Similarly, we established that EVA foam was safe for skin contact, but favored the closed-cell EVA over its' open-cell counterpart - which is absorbent - and evaluated its compatibility with common sterilization and reprocessing techniques $(70 \%$ isopropanol wipes, ionized hydrogen peroxide ${ }^{70}$ ).

\section{Stage 3: Regulatory approval and Implementation}

Regulatory approval:

Face shields are an FDA regulated Class I 510(k) exempt device ${ }^{68}$ making them appropriate to attempt to fabricate locally. Additional information on the FDA regulation of face shield products during the COVID-19 pandemic can be found in Table 1.

Clinical Testing: 
To assess face shield usability and safety, a cohort of 97 physicians, physician assistants, emergency department technicians, environmental service staff, and other individuals with patientfacing roles were recruited to an IRB-approved study from the Emergency Department at Brigham and Women's Hospital. Users wore the face shield during their shifts and completed a questionnaire on baseline experiences and attitudes. A majority of users indicated that they had a better experience with the PanFab face shield as compared to the hospital standard-issue, disposable model. This data was then presented to the appropriate stakeholders at BWH, and the product received approval for use as part of a clinical workflow.

\section{Production and Implementation:}

Following validation of the final design through an IRB-approved study at BWH hospital, over 3,000 face shields have been manufactured and deployed to meet local demand. These face shields were produced via distributed rapid manufacturing in collaboration with a local makerspace community (BoroBot), Salesforce, a small-scale prototyping company (SunPe Prototype), largescale manufacturers of non-medical supplies (iRobot and Velcro), in addition to academic partners (Harvard Graduate School of Design, the Shin Laboratory at the Brigham and Women's Hospital's Stepping Strong Foundation for Trauma Innovation, and the Wentworth Institute of Technology). All design files were made openly available on the PanFab website and on NIH 3DPrint exchange platform to allow other manufacturers and locality to produce the face-shield.

and fabricators contributing to such efforts. Note that regulatory documents from $\mathrm{NIOSH}, \mathrm{ANSI}$, ISO and DIN typically cost several hundred USD but many are being made freely available for the duration of the current healthcare emergency.

1. Conduct a thorough needs assessment to understand the most significant problems, assess current demand and supply, supply chain issues and material availability, and tolerance for different styles and types of products. Perform this analysis by speaking to a broad range of 
stakeholders early in the process and make sure to consider diverse body types and clinical roles. Secure examples of existing products and assess their strengths and weaknesses. Download and review relevant NIOSH/ANSI or regulatory documents from other agencies.

2. Solicit end-user, expert, and clinical feedback early and often throughout the design process to ensure the product satisfies anticipated and unanticipated needs. Frequent assessment increases the likelihood of end-user buy-in, promotes adoption, and enables rapid adoption of new solutions in response to changing demands. Consulting with senior medical staff and division heads can be very helpful in this setting. An IRB-approved clinical protocol may be required, particularly if a formal survey is to be conducted.

3. Conduct a rigorous assessment of biocompatibility, sterilization and risk. For PPE-type devices, research issues related to biocompatibility, manufacturing, intended duration/frequency of use, and anatomic location. Use materials previously established to be safe for the intended use if at all possible - using available scientific literature, medical device standards, or other devices previously reviewed by FDA. Be aware of likely sterilization requirements and feasibility since not every method of sterilization is compatible with every device. Review ISO $14971^{51}$, AISO 10993 -1 and FDA's associated guidance, and ISO 18562-1 for additional information.

4. Search design repositories for suitable designs that can be used as-is or modified to meet local requirements and fabrication capabilities. Many online forums have emerged to assist in the dissemination of best practices. Publicly funded designs should be available under nonrestrictive licenses from resources such as the National Institutes of Health 3D Print Exchange ${ }^{4}$.

5. Consider manufacturing methods, such as 3D printing and laser cutting, which enable rapid prototyping, and low-volume manufacturing, while keeping in mind a possible transition to more scalable approaches (e.g., injection molding) for large scale manufacturing. Consider which types of equipment will be available for the duration of the project and whether you need to reach out to local shops for access to higher-end equipment. 
6. Avoid action bias, which results in premature action and the rapid development of potentially suboptimal or undesired solutions. Even in a crisis, it is important to proceed deliberately to ensure that products are usable, safe, and durable.

7. Develop a written process for device performance validation through measurement of fit-forpurpose and alignment with end-user needs and regulatory standards. Check with your target users regarding the testing requirements and IRB-based testing capabilities. Consider life cycle issues including whether products will be withdrawn from service at the end of a medical emergency.

8. Provide documentation. Make sure to include accurate and complete labels and product information as inserts with the final products; consider complementing this with QR codes and online resources to provide users with the most up to date information. Consider making new designs and any improvements on existing designs publically available.

\section{Conclusions and future prospects}

The COVID-19 pandemic has made clear the fragility of medical supply chains which resulted in rapid and severe supply shortages. The communities of designers, fabricators and healthcare professionals who have come together to supply locally made substitutes using rapid manufacturing methods have revealed a hitherto untapped capacity to make the provision of medical supplies more resilient. The great strength of community efforts is that they avoid extended product development cycles and bypass international lowest-cost supply chains in favor of rapid innovation and efficient execution. However, even in a crisis, the risks of using alternative, locally-manufactured medical devices must be carefully evaluated and mitigated. Hastily designed products made without appropriate stakeholder input are unlikely to be used clinically, representing a loss of time and resources by wellintentioned makers. If poorly designed devices make their way into clinical use, they can pose a substantial threat. This paper provides a framework for crisis-responsive medical product design that is intended to avoid these risks. The framework is informed by traditional FDA guidance on product 
approval, implementation, and validation, but adapts to constraints in time, material resources, and human capital.

What does the current state of the COVID-19 response portend for the future? A transition from purely local efforts to national and international collaborations is already underway. These initiatives include several focused on PPE including Get Us PPE ${ }^{71}$, Covid19 Masks ${ }^{72}$, Mask Match ${ }^{73}$, Project ${\mathrm{N} 95^{74} \text {, and PPELink }}^{75}$ and larger efforts such as Open Source Medical Supplies (OSMS) ${ }^{76}$, America Makes $^{77}$, and the NIH 3-D Print Exchange ${ }^{4}$. State governments and companies such as Gillette (a P\&G Company $)^{78}$, and Lovepop ${ }^{79}$, are coming together through initiatives like the Manufacturing Emergency Response Team (M-ERT) ${ }^{66}$ to design, develop, manufacture, and donate or sell thousands of devices. These consortia facilitate stakeholder interactions and device testing, operations that are hard to perform on individual bases. Continuing these efforts will build greater resilience for future pandemics.

A key unanswered question is what will happen to non-traditional medical devices and innovative new designs when the COVID-19 pandemic recedes. These innovative products will likely be abandoned in favor of the standardized lowest-cost alternatives. In response to MERS, SARs, influenza and other zoonotic transfers, the design deficiencies of existing products and the fragility of supply chains were addressed by a comprehensive series of government reports spanning two decades. A wide variety of potentially effective solutions were proposed ${ }^{63}$ by government and private entities but few proactive solutions were actually implemented. To avoid forgetting the lessons of COVID-19 pandemic, it is essential that innovative designs and approaches be further developed, tested, and integrated into normal regulatory chains so they are ready for future emergencies. Changes in regulatory policies would also enable a middle ground, balancing the rigidity of the pre-crisis approach with the free-for-all and counterfeiting ${ }^{80}$ unintentionally enabled by EUAs. We envision the development of open-source repositories, such as NIH 3D Print Exchange, to facilitate independent testing of products to meet key $\mathrm{NIOSH}, \mathrm{FDA}$ and other requirements for functionality and safety. These entities could also facilitate a set of prescriptive approaches and best practices geared to the capabilities of small-scale fabrications in place of formal QMS and SPC. Training materials for designers, fabricators 
and healthcare institutions should be also developed to help break down barriers to communication. Institutional changes should also include adding makers and engineers (potentially drawn from the existing medical staff) to incident command and procurement teams so that non-traditional designs can be more effectively vetted; model IRB protocols should be prepared for products that need clinical testing. Finally, patented designs should be placed in repositories for public use during health emergencies ${ }^{63}$ to avoid delays from patent disputes. Such improvements to our infrastructure will dramatically improve our ability to respond to future waves of the COVID-19 pandemic and other crises in the United States and across the globe.

\section{Acknowledgements}

Local citizens and engineers have generously donated their time and resources to PanFab and they are essential to program success. This work was supported by the Harvard-MIT Center for Regulatory Science and by NIH/NCl grants P30-CA006516, U54-CA225088 (to PKS, NL and DP) and by T32GM007753 (to DP) and by the Harvard Ludwig Center. AKC is supported by the Hugh Hampton Young Fellowship of MIT. MJA is a recipient of the Friends of McGovern Graduate Fellowship.

\section{Author Contributions}

Article conception: M.J.A, D.P., S.S., N.R.L., B.L.-E., P.K.S.

Writing: M.J.A, D.P., S.S., L.A., A.A., H.Y., J.F., M.S.S., B.L.-E., P.K.S.

Editorial feedback: M.J.A, D.P., S.S., L.A., A.A., H.Y., A.C., J.F., M.S.S., S.H.Y., N.R.L., B.L.-E., P.K.S. Greater Boston Pandemic Fabrication Team (PanFab) Consortium Coordination: D.P., H.Y., P.K.S.

\section{Declaration of Interests}

PKS is a member of the SAB or Board of Directors of Applied Biomath, Glencoe Software and RareCyte Inc and has equity in these companies. In the last five years the Sorger lab has received 
research funding from Novartis and Merck. PKS declares that none of these relationships are directly or indirectly related to the content of this manuscript. NRB is a consultant for or has received honoraria from the following companies: Seattle Genetics, Sanofi, and Bayer.

\section{Supplemental Information}

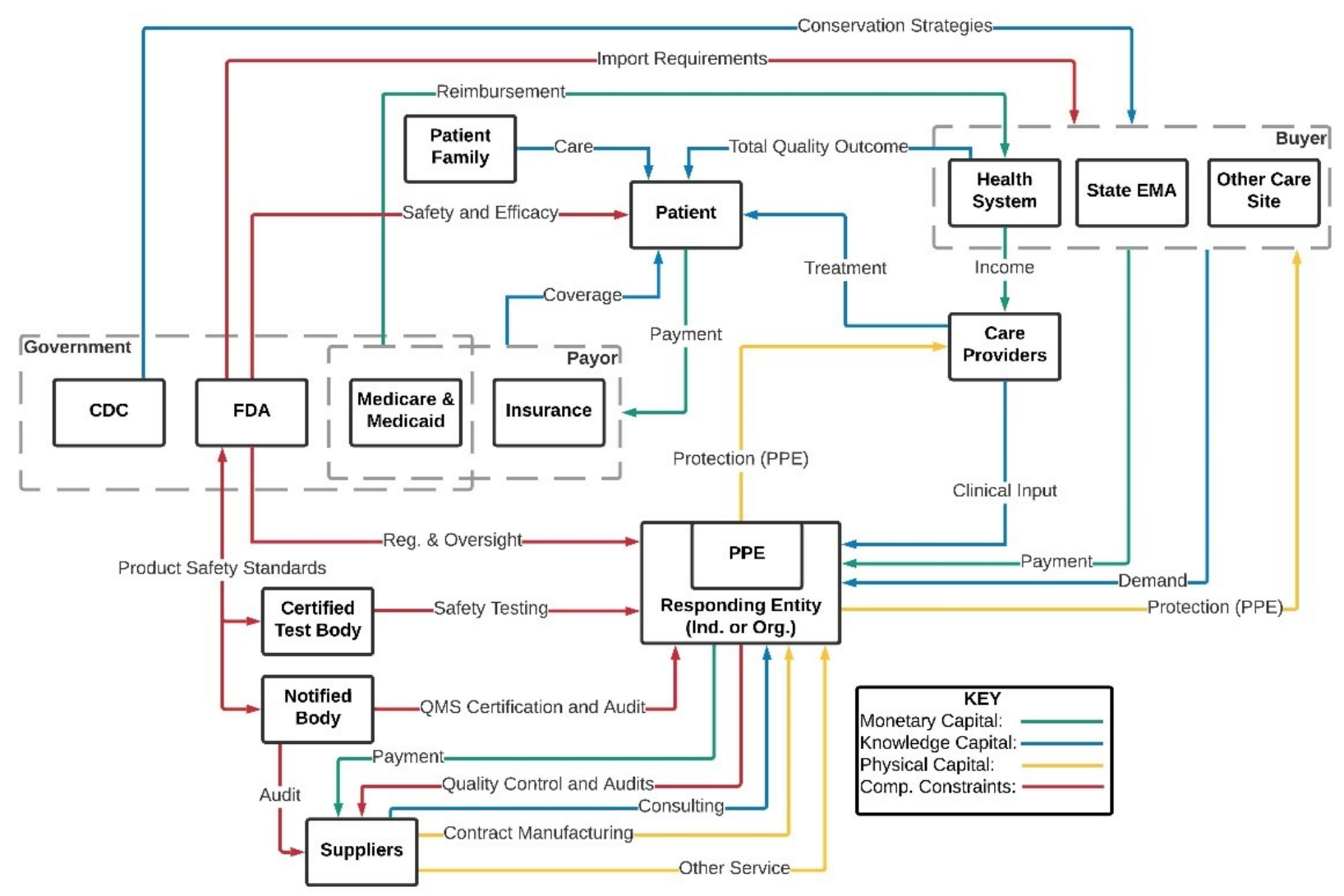

\section{Supplementary Figure S1: Stakeholder value network demonstrates the complex relationship} between all the stakeholders involved in the conventional medical device process. Related to Figure 1. Products scaling up must eventually consult and comply with the needs and regulations of all of these stakeholders. QMS: quality management system, Ind: Individual, Reg: regulatory, Org: organization. Comp: Compliance.

\section{References}


1. Shortage of personal protective equipment endangering health workers worldwide. $W H O$ https://www.who.int/news-room/detail/03-03-2020-shortage-of-personal-protective-equipmentendangering-health-workers-worldwide.

2. 3D Printing Medical Equipment in Response to the COVID-19 Pandemic. NIH Library https://www.nihlibrary.nih.gov/services/3d-printing-service/3d-printing-medical-equipment-response-covid19-pandemic (2020).

3. FAQs on 3D Printing of Medical Devices, Accessories, Components, and Parts During the COVID-19 Pandemic. U.S. Food and Drug Administration https://www.fda.gov/medical-devices/3d-printing-medicaldevices/faqs-3d-printing-medical-devices-accessories-components-and-parts-during-covid-19-pandemic (2020).

4. NIH 3D Print Exchange | A collection of biomedical 3D printable files and 3D printing resources supported by the National Institutes of Health (NIH). https://3dprint.nih.gov/.

5. Hanefeld, J. et al. Towards an understanding of resilience: responding to health systems shocks. Health Policy Plan. 33, 355-367 (2018).

6. Stevens, R. Why DIY 3D-Printed Face Masks and Shields Are So Risky. Slate Magazine https://slate.com/technology/2020/04/diy-3d-printed-face-masks-shields-coronavirus.html (2020).

7. Aitchison, G. A., Hukins, D. W. L., Parry, J. J., Shepherd, D. E. T. \& Trotman, S. G. A Review of the Design Process for Implantable Orthopedic Medical Devices. Open Biomed. Eng. J. 3, 21-27 (2009).

8. Alexander, K. \& Clarkson, P. J. A validation model for the medical devices industry. J. Eng. Des. 13, 197204 (2002).

9. Pietzsch, J. B., Shluzas, L. A., Paté-Cornell, M. E., Yock, P. G. \& Linehan, J. H. Stage-Gate Process for the Development of Medical Devices. J. Med. Devices 3, 021004 (2009).

10. Ogot, M. M. \& Kremer, G. Engineering design: a practical guide. (Trafford, 2004).

11. Medina, L. A., Kremer, G. E. O. \& Wysk, R. A. Supporting medical device development: a standard product design process model. J. Eng. Des. 24, 83-119 (2013). 
12. Pietzsch, J. B., Aquino, L. M., Yock, P. G., Paté-Cornell, M. E. \& Linehan, J. H. Review of U.S. Medical Device Regulation. J. Med. Devices 1, 283-292 (2007).

13. Winston W. Royce. Managing the Development of Large Software Systems. Tech. Pap. West. Electron. Show Conv. WesCon 1-9 (1970).

14. Agile Project Management in Product Development Projects - ScienceDirect. https://www.sciencedirect.com/science/article/pii/S1877042814021259.

15. Design control guidance for medical device manufacturers. U.S. Food and Drug Administration https://www.fda.gov/regulatory-information/search-fda-guidance-documents/design-control-guidancemedical-device-manufacturers (1997).

16. PanFab News - Latest Results. https://www.panfab.org/.

17. Hack the Pandemic - Copper 3D | Antibacterial 3D Printing. https://copper3d.com/hackthepandemic/.

18. Thingiverse.com. Mask Frame 'CEG Extreme' and Small n95 fitter (High Filtration with Halyard H600) by ctwiles. https://www.thingiverse.com/thing:4262131.

19. 3D Printed Face Mask---- No Worries on Mask Shortage and Coronavirus Infection.

https://creality.com/info/makers-guide-3d-printed-face-mask-no-worries-on-mask-shortage-and-virusinfection-i00248i1.html.

20. COVID-19 Response. Lowell Makes https://lowellmakes.com/covid-19-response/ (2020).

21. Sher, D. WASP shares open source processes for production of personalized PPE masks and helmets. $3 D$ Printing Media Network https://www.3dprintingmedia.network/personalized-ppe-mask/ (2020).

22. How to Make Bellus3D’s Face Mask Fitter. Bellus3D: High-quality 3D face scanning http://www.bellus3d.com/solutions/facemask.

23. McAvoy, M. et al. 3D Printed frames to enable reuse and improve the fit of N95 and KN95 respirators. http://medrxiv.org/lookup/doi/10.1101/2020.07.20.20151019 (2020) doi:10.1101/2020.07.20.20151019.

24. Attaran, M. The rise of 3-D printing: The advantages of additive manufacturing over traditional manufacturing. Bus. Horiz. 60, 677-688 (2017). 
25. Technical Considerations for Additive Manufactured Medical Devices Guidance for Industry and Food and Drug Administration Staff. U.S. Food and Drug Administration https://www.fda.gov/regulatoryinformation/search-fda-guidance-documents/technical-considerations-additive-manufactured-medicaldevices (2017).

26. Dimitroulis, G., Austin, S., Sin Lee, P. V. \& Ackland, D. A new three-dimensional, print-on-demand temporomandibular prosthetic total joint replacement system: Preliminary outcomes. J. Cranio-Maxillo-fac. Surg. Off. Publ. Eur. Assoc. Cranio-Maxillo-fac. Surg. 46, 1192-1198 (2018).

27. Popescu, D. \& Laptoiu, D. Rapid prototyping for patient-specific surgical orthopaedics guides: A systematic literature review. Proc. Inst. Mech. Eng. [H] 230, 495-515 (2016).

28. Ganguli, A. et al. 3D printing for preoperative planning and surgical training: a review. Biomed. Microdevices 20, 65 (2018).

29. George, M., Aroom, K. R., Hawes, H. G., Gill, B. S. \& Love, J. 3D Printed Surgical Instruments: The Design and Fabrication Process. World J. Surg. 41, 314-319 (2017).

30. Oliveira, T. T. \& Reis, A. C. Fabrication of dental implants by the additive manufacturing method: A systematic review. J. Prosthet. Dent. 122, 270-274 (2019).

31. Prusa Face Shield. PrusaPrinters https://www.prusaprinters.org/prints/25857-prusa-face-shield.

32. Mostaghimi, A. et al. Regulatory and Safety Considerations in Deploying a Locally Fabricated, Reusable Face Shield in a Hospital Responding to the COVID-19 Pandemic. Med S2666634020300088 (2020) doi:10.1016/j.medj.2020.06.003.

33. BIDMC-led clinical trial identifies four novel 3D-printed swabs for use in COVID-19 testing. https://www.bidmc.org/about-bidmc/news/2020/04/3d-printed-swabs.

34. Callahan, C. J. et al. Open Development and Clinical Validation of Multiple 3D-Printed Sample-Collection Swabs: Rapid Resolution of a Critical COVID-19 Testing Bottleneck. http://medrxiv.org/lookup/doi/10.1101/2020.04.14.20065094 (2020) doi:10.1101/2020.04.14.20065094.

35. Home | COVID Swabs. https://printedswabs.org/.

36. Prisma Health introduces VESper. Prisma Health https://www.prismahealth.org/vesper/. 
37. Open Source COVID19 Medical Supply Guide. Google Docs https://docs.google.com/document/d/171FJTml1Q1kjSDLP0EegMERjg_0kk_7UfaRE4r66Mg/edit?usp=sharing\&usp=embed_facebook.

38. Single-Use Devices | Disinfection \& Sterilization Guidelines | Guidelines Library | Infection Control | CDC. https://www.cdc.gov/infectioncontrol/guidelines/disinfection/reuse-of-devices.html (2019).

39. Enforcement Priorities for Single-Use Devices Reprocessed by Third Parties and Hospitals. U.S. Food and Drug Administration https://www.fda.gov/regulatory-information/search-fda-guidancedocuments/enforcement-priorities-single-use-devices-reprocessed-third-parties-and-hospitals (2000).

40. List N: Disinfectants for Use Against SARS-CoV-2. US EPA https://www.epa.gov/pesticide-registration/listn-disinfectants-use-against-sars-cov-2 (2020).

41. Coronavirus Disease 2019 (COVID-19). Decontamination and Reuse of Filtering Facepiece Respirators. Centers for Disease Control and Prevention https://www.cdc.gov/coronavirus/2019-ncov/hcp/ppestrategy/face-masks.html (2020).

42. US Food and Drug Administration. Emergency Use Authorization; ASP STERRAD Sterilization Systems. (2020).

43. US Food and Drug Administration. Emergency Use Authorization; Battelle Critical Care Decontamination System. (2020).

44. US Food and Drug Administration. Emergency Use Authorization; Steris Corporation. (2020).

45. Use of International Standard ISO 10993-1, 'Biological evaluation of medical devices - Part 1: Evaluation and testing within a risk management process'. U.S. Food and Drug Administration https://www.fda.gov/regulatory-information/search-fda-guidance-documents/use-international-standard-iso10993-1-biological-evaluation-medical-devices-part-1-evaluation-and (2019).

46. Biocompatibility Assessment. U.S. Food and Drug Administration https://www.fda.gov/medicaldevices/cdrh-research-programs/biocompatibility-assessment (2018).

47. 14:00-17:00. ISO 10993-1:2018. ISO https://www.iso.org/cms/render/live/en/sites/isoorg/contents/data/standard/06/89/68936.html. 
48. ISO 18562-1:2017(en), Biocompatibility evaluation of breathing gas pathways in healthcare applications Part 1: Evaluation and testing within a risk management process. https://www.iso.org/obp/ui\#iso:std:iso:18562:-1:ed-1:v1:en.

49. 14:00-17:00. ISO 13485:2016. ISO https://www.iso.org/cms/render/live/en/sites/isoorg/contents/data/standard/05/97/59752.html.

50. Prusa Face Shield. PrusaPrinters https://www.prusaprinters.org/prints/25857-prusa-face-shield (2020).

51. Speer, J. \& Rish, T. ISO 14971 RISK MANAGEMENT FOR MEDICAL DEVICES: THE DEFINITIVE GUIDE. 41.

52. U.S. Department of Health \& Human Services. Secretary Azar Declares Public Health Emergency for United States for 2019 Novel Coronavirus. HHS.gov https://www.hhs.gov/about/news/2020/01/31/secretary-azar-declares-public-health-emergency-us-2019novel-coronavirus.html (2020).

53. Emergency Use Authorizations. U.S. Food and Drug Administration https://www.fda.gov/medicaldevices/emergency-situations-medical-devices/emergency-use-authorizations (2020).

54. US Food and Drug Administration. Emergency Use Authorization; Manufacturers of Face Shields. (2020).

55. US Food and Drug Administration. Emergency Use Authorization; Respirators. (2020).

56. Classify Your Medical Device. U.S. Food and Drug Administration https://www.fda.gov/medicaldevices/overview-device-regulation/classify-your-medical-device (2020).

57. Learn if a Medical Device Has Been Cleared by FDA for Marketing. U.S. Food and Drug Administration https://www.fda.gov/medical-devices/consumers-medical-devices/learn-if-medical-device-has-beencleared-fda-marketing (2018).

58. Safety and Performance Based Pathway Guidance for Industry and Food and Drug Administration. U.S. Food and Drug Administration https://www.fda.gov/regulatory-information/search-fda-guidancedocuments/safety-and-performance-based-pathway (2019).

59. Neyman, G. \& Irvin, C. B. A Single Ventilator for Multiple Simulated Patients to Meet Disaster Surge. Acad. Emerg. Med. 13, 1246-1249 (2006). 
60. Denise M. Hinton, Chief Scientist. Food and Drug Administration Emergency Use Authorization issued in response to concerns relating to insufficient supply and availability of FDA-cleared ventilators for use in healthcare settings to treat patients during the COVID-19 pandemic. (2020).

61. Joint Statement on Multiple Patients Per Ventilator. https://www.asahq.org/about-asa/newsroom/newsreleases/2020/03/joint-statement-on-multiple-patients-per-ventilator (2020).

62. Medical Device Exemptions 510(k) and GMP Requirements. U.S. Food and Drug Administration https://www.accessdata.fda.gov/scripts/cdrh/cfdocs/cfpcd/315.cfm.

63. Sinha, M. S., Bourgeois, F. T. \& Sorger, P. K. Personal Protective Equipment for COVID-19: Distributed Fabrication and Additive Manufacturing. Am. J. Public Health 110, 1162-1164 (2020).

64. MGB Center for COVID Innovation. https://covidinnovation.partners.org/.

65. N95DECON. N95DECON https://www.n95decon.org.

66. The Mass. Manufacturing Community Responds to COVID-19 | MassTech. https://masstech.org/M-ERT.

67. Coronavirus Disease 2019 (COVID-19). Interim Infection Prevention and Control Recommendations for Patients with Suspected or Confirmed Coronavirus Disease 2019 (COVID-19) in Healthcare Settings. Centers for Disease Control and Prevention https://www.cdc.gov/coronavirus/2019-ncov/hcp/infectioncontrol-recommendations.html (2020).

68. Roberge, R. J. Face shields for infection control: A review. J. Occup. Environ. Hyg. 13, 235-242 (2016).

69. Coronavirus Disease 2019 (COVID-19). Strategies for Optimizing the Supply of Eye Protection. Centers for Disease Control and Prevention https://www.cdc.gov/coronavirus/2019-ncov/hcp/ppe-strategy/eyeprotection.html (2020).

70. Cramer, A. et al. Analysis of SteraMist ionized hydrogen peroxide technology as a method for sterilizing N95 respirators and other personal protective equipment.

http://medrxiv.org/lookup/doi/10.1101/2020.04.19.20069997 (2020) doi:10.1101/2020.04.19.20069997.

71. \#GetUsPPE - Getting Protective Equipment to our Healthcare Heroes. Get Us PPE https://getusppe.org/.

72. COVID19Masks: A PPE exchange. Helping those who help others.

https://covid19masks.info/webapps/f?p=2222:1. 
73. Mask Match. Mask Match https://www.mask-match.com.

74. The National COVID-19 Critical Equipment Clearinghouse. Project N95 https://www.projectn95.org/.

75. PPE Link. PPE Link https://ppelink.wordpress.com/.

76. Open Source Medical Supplies. https://opensourcemedicalsupplies.org/.

77. America Makes - National Additive Manufacturing Innovation Institute. America Makes https://www.americamakes.us/.

78. Chesto, J. P\&G is making tens of thousands of face shields at Gillette plant in Southie - The Boston Globe. BostonGlobe.com https://www.bostonglobe.com/2020/04/13/business/pg-is-making-tens-thousands-faceshields-gillette-plant-southie/.

79. Walrath, R. Lovepop begins manufacturing personal protective equipment. AmericanInno https://www.americaninno.com/boston/inno-news-boston/3d-card-startup-lovepop-begins-manufacturingprotective-equipment/ (2020).

80. Plana, D. et al. Assessing the quality of nontraditional N95 filtering face-piece respirators available during the COVID-19 pandemic. (2020) doi:10.1101/2020.07.25.20161968.

81. Product Classification. U.S. Food and Drug Administration https://www.accessdata.fda.gov/scripts/cdrh/cfdocs/cfPCD/classification.cfm?ID=FXX.

82. Enforcement Policy for Face Masks and Respirators During the Coronavirus Disease (COVID-19) Public Health Emergency (Revised). U.S. Food and Drug Administration https://www.fda.gov/regulatoryinformation/search-fda-guidance-documents/enforcement-policy-face-masks-and-respirators-duringcoronavirus-disease-covid-19-public-health (2020).

83. Part 84-approval of respiratory protective devices. Electronic Code of Federal Regulations vol. §84.174 Filter efficiency level determination test-non-powered series $N, R$, and $P$ filtration.

84. Approval Tests and Standards for Air-Purifying Particulate Respirators. Federal Register https://www.federalregister.gov/documents/2020/04/14/2020-07804/approval-tests-and-standards-for-airpurifying-particulate-respirators (2020). 
85. Medical Devices; Exemption From Premarket Notification: Class II Devices; Surgical Apparel. Federal Register https://www.federalregister.gov/documents/2018/05/17/2018-10563/medical-devices-exemptionfrom-premarket-notification-class-ii-devices-surgicalapparel?fbclid=IwAR0p6t1z5BttFbyGIK6Krch565d0Y96UKaulqrXcDtqJbnntRBg0F7HtfeQ (2018).

86. Ouellette, L. L. Written Description: Regulatory Responses to N95 Respirator Shortages. Written Description https://writtendescription.blogspot.com/2020/04/regulatory-responses-to-n95-respirator.html (2020). 